\title{
A novel nonsense SMC1A mutation in a patient with intractable epilepsy and cardiac malformation
}

\author{
Yasutsugu Chinen (1]', Sadao Nakamura', Takuya Kaneshi', Mami Nakayashiro², Kumiko Yanagi ${ }^{3}$, Tadashi Kaname (0) ${ }^{3}$, \\ Kenji Naritomi ${ }^{4}$ and Koichi Nakanishi id
}

\begin{abstract}
Cornelia de Lange syndrome (CdLS) is a cohesinopathy caused by genetic variations. We present a female with SMC1A-associated CdLS with a novel SMC1A truncation mutation (p. Arg499Ter), transposition of the great arteries, and periodic intractable seizures from 40 months of age. A review of the literature revealed that a seizure-free period after birth of at least 15 months is required for these patients to be able to walk, irrespective of the epileptic course.
\end{abstract}

Cornelia de Lange syndrome (CdLS) [MIM: 122470, 300590, 300882, 610759, and 614701] is a congenital multisystemic disorder with widely varied characteristics ranging from mild (nonclassical phenotype) to severe (classical phenotype) that is caused by genetic variants of structural or regulatory components of the cohesin complex $^{1,2}$. Classical CdLS is caused by mutations in $N I P B L$, while nonclassical CdLS is caused by mutations in $S M C 1 A, S M C 3 A, R A D 21$, and $H D A C 8^{1}$. Missense variants and small in-frame deletions in $S M C 1 A$, located at Xp11.22, account for $\sim 5 \%$ of CdLS cases. A review of the literature revealed 60 cases of SMC1A-associated CdLS with a male-to-female ratio of $1: 2^{3}$. A total of nine cases of CdLS with congenital cardiac defects (CHD) have been reviewed ${ }^{4}$, although the incidence of CHD in patients with CdLS is $\sim 30 \%^{5}$. SMC1A-related CdLS arises from a dominant negative effect in females ${ }^{2}$. Females with SMC1A mutations leading to protein truncation are affected by intractable epilepsy, severe developmental retardation, and few craniofacial differences ${ }^{4,6}$. Age at

Correspondence: Yasutsugu Chinen (ychinen@med.u-ryukyu.ac.jp)

${ }^{1}$ Department of Child Health and Welfare (Pediatrics), Graduate School of Medicine, University of the Ryukyus, Nishihara, Japan

2Department of Pediatrics, Okinawa Prefectural Nanbu Medical Center Children's Medical Center, Haebaru, Okinawa, Japan

Full list of author information is available at the end of the article. presentation with first epileptic seizures ranges from $<1$ month to 17 months ${ }^{6}$.

In our case of CdLS with an SMC1A truncating mutation, the female patient was the second child born to a healthy, nonconsanguineous couple when her mother and father were 35 and 42 years of age, respectively. There was no family history of CdLS. She was born via emergency cesarean section at 35 weeks gestation due to fetal distress. At birth, her weight was $1636 \mathrm{~g}(-1.9 \mathrm{SD})$, length was $43.5 \mathrm{~cm} \quad(-0.43 \mathrm{SD})$, and occipitofrontal circumference (OFC) was $30.2 \mathrm{~cm}(-0.6 \mathrm{SD})$. She had been hospitalized in the neonatal intensive care unit for 3 months because of failure to thrive and transposition of the great arteries (TGA) type III congenital heart defects with a ventricular septal defect and pulmonary artery stenosis. At 11 months of age, the Blalock-Taussig shunt operation was performed, followed by home oxygen therapy. At 1 year 10 months of age, the Nissen operation was performed for gastroesophageal reflux disease. At 3 years 4 months of age, tonic seizures emerged. Hypoglycemia and high ammonia levels appeared. Hyperammonemia $(405 \mu \mathrm{g} / \mathrm{dl}$; normal range: $36-86 \mu \mathrm{g} / \mathrm{dl})$ and repetitive hypoglycemia occurred after she suffered from bacterial pneumonia at 4 years 7 months of age. She had been taking six meals a day, two with cornstarch. She was referred to our clinic for detailed examination of 
metabolic diseases. At 4 years 9 months, she weighed $14.1 \mathrm{~kg}(-1.3 \mathrm{SD})$, her height was $100.5 \mathrm{~cm}(-1.1 \mathrm{SD})$, and her OFC was $46.8 \mathrm{~cm}(-2.2 \mathrm{SD})$. She had a prominent forehead, hypertelorism, thick eyebrows, broad nasal tip, depressed nasal bridge, full cheeks, left cupped ear, right prominent antihelix, high-arched palate, thin upper lip, crowded teeth, slender fingers, left talipes varus, and left second short toe (Fig. 1a-g). The results of all biochemical tests and gas chromatography/mass spectrometry (GC/ MS) of urine and tandem mass spectrometry (MS/MS) of dry blood spots were normal. Hypoglycemia was observed during hospitalization, and occasionally episodes caused hypoglycemia irrespective of meal time. Following this, there were no episodes of hypoglycemia for 3 years. A fasting test was conducted at 7 years 3 months of age. Hypoglycemia $(37 \mathrm{mg} / \mathrm{dl}$; normal range $70-105 \mathrm{mg} / \mathrm{dl}$ ) appeared $17 \mathrm{~h}$ after the last meal, but there were no findings of coldness, lethargy, hyperammonemia, or metabolic acidosis. Additionally, all urine GC/MS and dry blood spot MS/MS results were normal. Magnetic resonance imaging of the brain revealed normal findings. She had left sensorineural deafness $(100 \mathrm{~dB})$. Her developmental milestones were delayed: head control at 6 months of age, rollover at 6 months, sitting unaided at 2 years and 4 months, and walking at 2 years and 5 months. At 15 years 2 months of age, she was unable to speak meaningful words, her height was $133.0 \mathrm{~cm}(-4.6 \mathrm{SD})$, and her weight was $27.1 \mathrm{~kg}(-3.0 \mathrm{SD})$. Her menstruation started regularly at 14 years 9 months of age. At 13 years of age, six persistent deciduous teeth with no dental caries were extracted.

Three-day-long continuous intractable generalized tonic seizures (GTS) occurred every 2 weeks from 3 years to 4 months of age. At 15 years of age, moderately beneficial antiepileptic drugs (AEDs) were lamotrigine (LMT) and levetiracetam (LEV). AEDs tried without success were clobazam $(\mathrm{CLB})$, potassium bromide $(\mathrm{KBr})$, and carbamazepine (CBZ). She had a Jatene operation for TGA type III at 5 years 2 months of age and corrective surgery for the left talipes varus at 6 years 10 months. Chromosomal analysis showed a normal karyotype. We searched the original computerized database for possible malformation syndromes: UR-DBMS (University of the RyukyusDatabase for Malformation Syndromes: http:// becomerich.lab.u-ryukyu.ac.jp) edited by Naritomi ${ }^{7}$. Suggested candidates matching over 12 signs were 4pter-p13 trisomy and Xpter-p21 monosomy, 13q14-qter trisomy and 5pter-p13 monosomy, CDLS1 (MIM 122470), CDLS3 (MIM 610759), Noonan syndrome 1 (NS1) (MIM 163950), 1q21.1 deletion syndrome, 12p trisomy, and
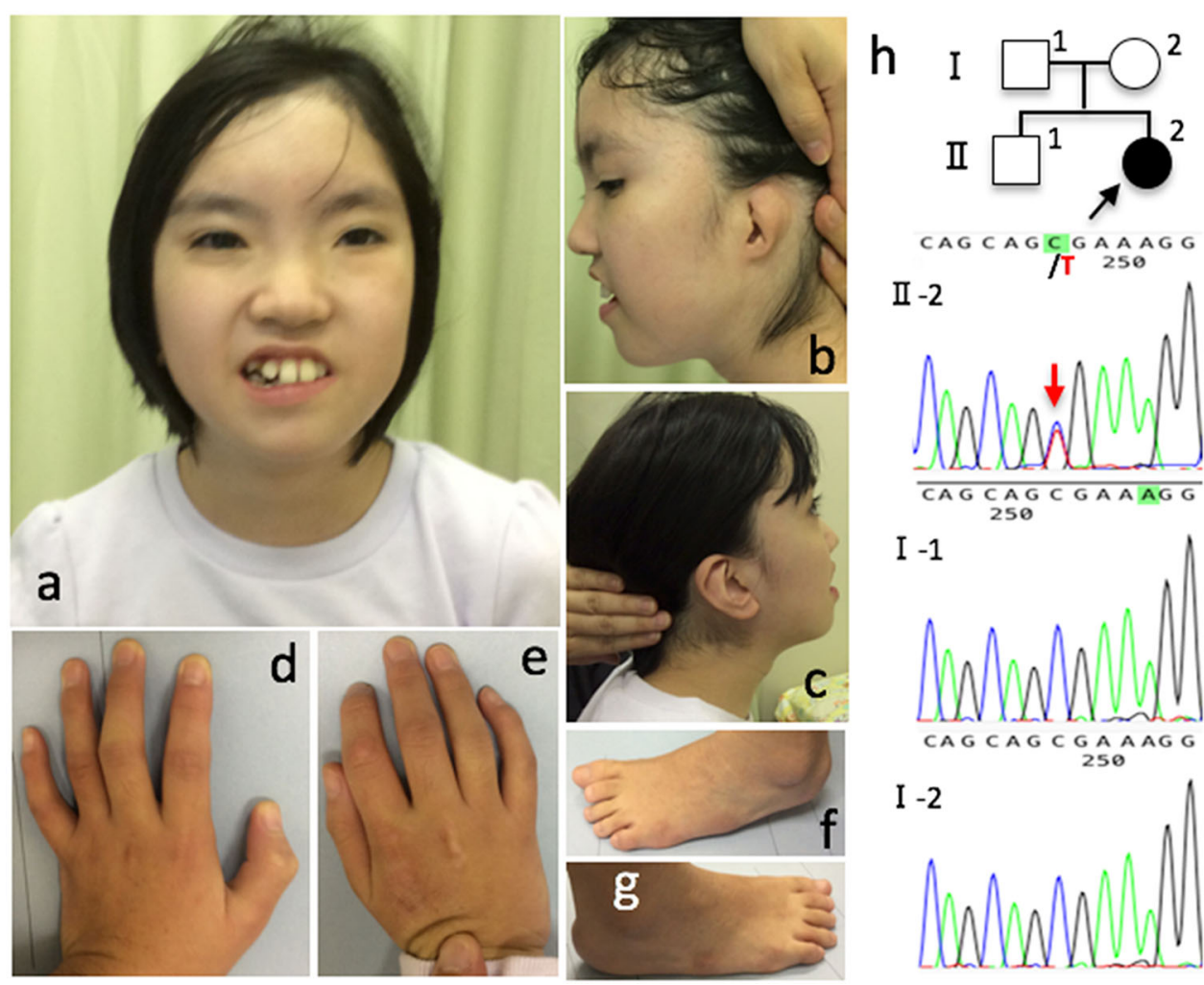

II -2
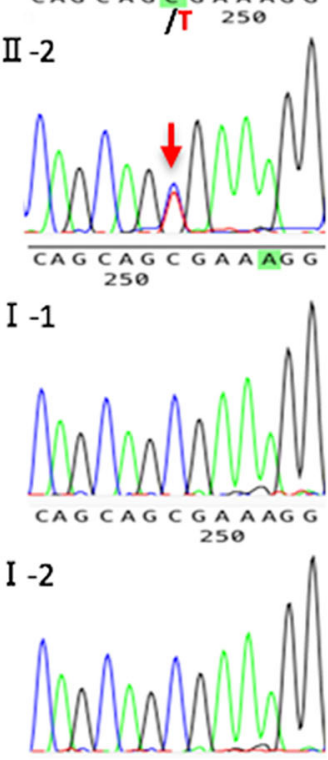

Fig. 1 Patient's appearance at 12 years of age. Face (front, a; lateral, $\mathbf{b}$ and $\mathbf{c}$ ), hand (left, $\mathbf{d}$; right $\mathbf{e}$ ), and foot (left, f; right, $\mathbf{g}$ ). Sequencing profiles of the SMC1A gene of the patient and her parents are shown (h) 


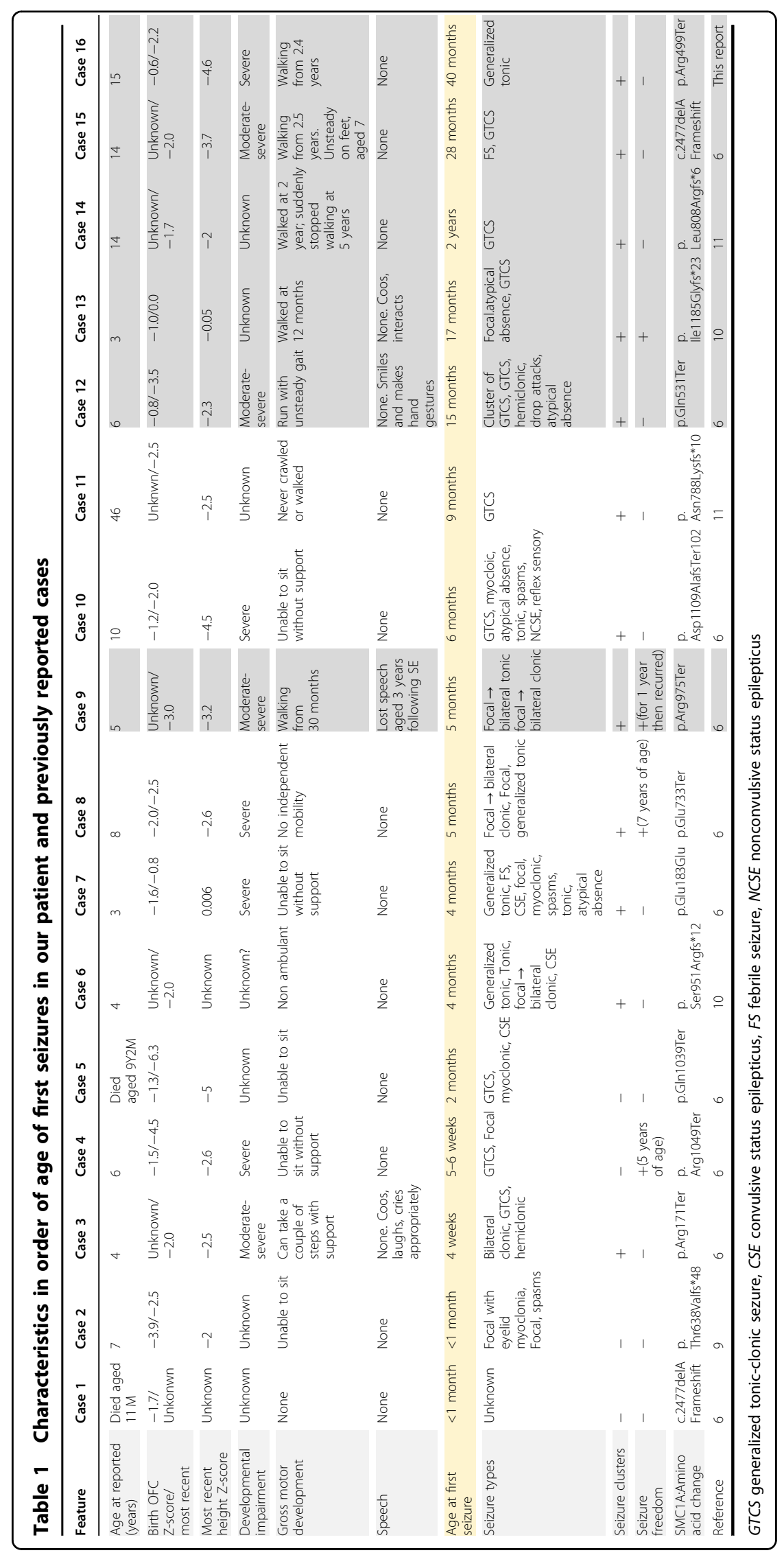


16p11.2 deletion syndrome. Whole-exome sequencing was performed using the SureSelect Human All Exon V6 Kit (Agilent Technologies, Santa Clara, CA) and HiSeq2500 (Illumina, San Diego, CA). To identify diseasecausing mutations, we excluded all known variants found in the 1000 Genomes database (http://www. internationalgenome.org/), Japanese Genomes database ${ }^{8}$, dbSNP (http://www.ncbi.nlm.gov/SNP), the genome Aggregation Database (gnomAD; http://gnomad. broadinstitute.org/), and the Human Genetic Variation Database (HGVD; http://www.genome.med.kyoto-u.ac.jp/ SnpDB/). Heterozygous SMC1A (NM_006306) mutations cause CdLS, the symptoms of which fit with those of our patient. We identified a heterozygous single nucleotide variation (c. C1495T) in SMC1A exon 9 that results in a nonsense mutation (p. Arg499Ter) and a truncated protein. The p. Arg499Ter variant was not detected in her parents, suggesting that the variant was de novo. This was confirmed by Sanger sequencing (Fig. 1h). This is the first description of the p. Arg499Ter variant. Mutation Taster (http://www.mutationtaster.org) predictions indicate that this is a disease-causing variant. This study was performed in accordance with the standards of the Ethics Committee of the Ryukyus Graduate School of Medicine (Okinawa, Japan). Informed consent was obtained from her parents by Dr. Yasutsugu Chinen.

Our patient with $S M C 1 A$-associated CdLS had a nonclassical CdLS type. This was determined using the clinical CdLS scoring system, which consists of 9 points, including three cardinal and three suggestive features ${ }^{1,6}$. Her first seizures, which occurred at 40 months of age, were the latest onset recorded. Seizure onset occurred much earlier in the 15 previously reported patients with protein truncating mutations in $S M C 1 A^{6}$. When these cases, including the one presented here, were arranged in order of age of first seizures, we observed that only one (9.1\%) of the 11 patients who had their first seizure at less than 9 months of age could walk. In contrast, all of the patients (100\%) who had their first seizure after 15 months could walk. This observation was independent of the type of epilepsy and the truncated amino acid position in SMC1A (Table 1, modified from Symonds et al. 2017 $)^{6,9-11}$. Although the number of cases is small, this observation suggests a correlation between age of epilepsy onset and independent walking. If the first seizures develop before the patient could walk, walking without assistance may be difficult. Only one patient, case 9, developed seizures in the 5 months after birth and was able to walk at 30 months of age. This patient was seizurefree for 1 year after commencement of levetiracetam treatment, but the seizures recurred. In case 4 , seizures started from 5-6 weeks of age and stopped occurring at 5 years of age after commencement of phenobarbitone treatment and adoption of a ketogenic diet. In case 8 , seizures started from 5 months of age and stopped occurring at 7 years of age after commencement of gabapentin treatment and adoption of a ketogenic diet. Taken together, these cases indicate that at least the first 15 months after birth needs to be seizure-free for these patients to walk, irrespective of the epileptic course.

CHD with $S M C 1 A$-associated CdLS has been previously reported in 13 cases, including ours, yet this is the first report including a TGA diagnosis (Supplementary Table $1)^{2-4,6,12-18}$. In patients with CHD, the rate of substitution or mutation at an arginine position is $42 \%(5 / 12)$, and in patients without $\mathrm{CHD}$, the rate is $50 \%(11 / 22)$. The posttranslational modification of proteins by arginine methylation is functionally important in the SMC1A protein ${ }^{19}$. Dysregulation of cohesin by the SMC1A protein may cause CHD in a zebrafish cohesinopathy model ${ }^{20}$. The same variations at arginine positions, including p. Val58Arg62del, p. Arg196, p. Arg496, and p. Arg693, revealed discordant heart defects. However, there may be no relationship between SMC1A arginine substitution and $\mathrm{CHD}$, and we were unable to show whether such SMC1A variations affect arginine methylation.

Our patient's episodes of hypoglycemia showed no remarkable causal disease and were considered clinical symptoms caused by dumping syndrome. However, we could not regularly observe reproducible events. The fasting test at 7 years 3 months of age revealed a normal reaction without hypoglycemic episodes. Infancy is considered to be a time period of low glycogen storage corresponding to age. If hypoglycemia occurs during this period, further careful investigation and dietary adjustments might be necessary.

\section{Acknowledgements \\ We are indebted to the patient and her parents, nurses, and physicians who supported this study. We thank Rebecca Porter, PhD, from Edanz Group (www. edanzediting.com/ac) for editing a draft of this manuscript. This work was supported in part by a grant from the Initiative on Rare and Undiagnosed Diseases in Pediatrics (IRUD-P) project (16ek0109166h0002) from the Japan Agency for Medical Research and Development (AMED).}

\section{Author details \\ ${ }^{1}$ Department of Child Health and Welfare (Pediatrics), Graduate School of Medicine, University of the Ryukyus, Nishihara, Japan. '2Department of Pediatrics, Okinawa Prefectural Nanbu Medical Center Children's Medical Center, Haebaru, Okinawa, Japan. ${ }^{3}$ Department of Genome Medicine, National Center for Child Health and Development, Tokyo, Japan. ${ }^{4}$ Okinawa Nanbu Habilitation and Medical Center, Naha, Japan}

\section{HGV Database}

The relevant data from this Data Report are hosted at the Human Genome Variation Database at https://doi.org/10.6084/m9.figshare.hgv.2570.

Conflict of interest

The authors declare that they have no conflict of interest.

\section{Publisher's note}

Springer Nature remains neutral with regard to jurisdictional claims in published maps and institutional affiliations. 
Supplementary Information is available for this paper at https://doi.org/ 10.1038/s41439-019-0053-y.

Received: 16 February 2019 Revised: 20 March 2019 Accepted: 31 March 2019.

Published online: 13 May 2019

\section{References}

1. Kline A. D., et al. Diagnosis and management of Cornelia de Lange syndrome: first international consensus statement. Nat. Rev. Genet. https://doi.org/ 10.1038/s41576-018-0031-0 (2018).

2. Liu, J. et al. SMC1A expression and mechanism of pathogenicity in probands with X-Linked Cornelia de Lange syndrome. Hum. Mutat. 30, 1535-1542 (2009).

3. Gervasini, C. et al. Cornelia de Lange individuals with new and recurrent SMC1A mutations enhance delineation of mutation repertoire and phenotypic spectrum. Am. J. Med. Genet. A 161, 2909-2919 (2013).

4. Wenger, T. L. et al. Novel findings of left ventricular non-compaction cardiomyopathy, microform cleft lip and poor vision in patient with SMC1A- associated Cornelia de Lange syndrome. Am. J. Med. Genet. A. 173, 414-420 (2017).

5. Selicorni, A. et al. Analysis of congenital heart defects in 87 consecutive patients with Brachmann-de Lange syndrome. Am. J. Med. Genet. A. 149, 1268-1272 (2009).

6. Symonds, J. D. et al. Heterozygous truncation mutations of the SMC1A gene cause a severe early onset epilepsy with cluster seizures in females: detailed phenotyping of 10 new cases. Epilepsia. 58, 565-575 (2017).

7. Naritomi, K. Application for an original computerized database (UR-DBMS) for diagnosis of the malformation syndromes. Cong. Anom. 38, 251-258 (1988).

8. Nagasaki, M. et al. Rare variant discovery by deep whole-genome sequencing of 1,070 Japanese individuals. Nat. Commun. 21, 8018 (2015).
9. Lebrun, N. et al. Early-onset encephalopathy with epilepsy associated with a novel splice site mutation in SMC1A. Am. J. Med. Genet. A 167, 3076-3081 (2015).

10. Goldstein, J. H. et al. Novel SMC1A frameshift mutations in children with developmentaldelay and epilepsy. Eur. J. Med. Genet. 58, 562-568 (2015).

11. Jansen, S. et al. De novo loss-of-function mutations in X-linked SMC1A cause severe ID and therapy-resistant epilepsy in females: expanding the phenotypic spectrum. Clin. Genet. 90, 413-419 (2016).

12. Deardorff, M. A. et al. Mutations in cohesin complex members SMC3 and SMC1A cause a mild variant of cornelia de lange syndrome with predominant mental retardation. Am. J. Hum. Genet. 80, 485-494 (2007).

13. Limongelli, G. et al. Hypertrophic cardiomyopathy in a girl with Cornelia de Lange syndrome due to mutation in SMC1A. Am. J. Med. Genet. A. 152 2127-2129 (2010).

14. Mannini, L., Cucco, F., Quarantotti, V., Krantz, I. D. \& Musio, A. Mutation spectrum and genotype-phenotype correlation in Cornelia de Lange syndrome. Hum. Mutat. 34, 1589-1596 (2013).

15. Pié, J. et al. Mutations and variants in the cohesion factor genes NIPBL, SMC1A, and SMC3 in a cohort of 30 unrelated patients with Cornelia de Lange syndrome. Am. J. Med. Genet. A 152, 924-929 (2010).

16. Musio, A. et al. X-linked Cornelia de Lange syndrome owing to SMC1L1 mutations. Nat. Genet. 38, 528-530 (2006).

17. Mannini, L., Liu, J., Krantz, I. D. \& Musio, A. Spectrum and consequences of SMC1A mutations: the unexpected involvement of a core component of cohesin in human disease. Hum. Mutat. 31, 5-10 (2010).

18. Borck, G. Incidence and clinical features of X-linked Cornelia de Lange syndrome due to SMC1L1 mutations. Hum. Mutat. 28, 205-206 (2007).

19. Larsen, S. C. et al. Proteome-wide analysis of arginine monomethylation reveals widespread occurrence in human cells. Sci. Signal. 9, 443 (2016).

20. Schuster, K. et al. A neural crest origin for cohesinopathy heart defects. Hum. Mol. Genet. 24, 7005-7016 (2015). 